\title{
Letter to the Editor - Open Bite Malocclusion: Analysis of the Underlying Components
}

\author{
Heba E. Akl ${ }^{1}$, Amira A. Aboalnaga ${ }^{2}$ and Yehya A. Mostafa ${ }^{3 *}$ \\ ${ }^{1}$ Demonstrator, Department of Orthodontics, Faculty of Dentistry, Cairo University, Cairo, Egypt \\ ${ }^{2}$ Lecturer, Department of Orthodontics and Dentofacial Orthopedics, Faculty of Dentistry, Cairo University, Cairo, Egypt \\ ${ }^{3}$ Professor and Ex-Chairperson, Department of Orthodontics, Faculty of Dentistry, Cairo University, Egypt
}

${ }^{\star}$ Corresponding author: Yehya A. Mostafa DDS, FDSRCSED, MS, PHD, Professor and Ex-Chairperson, Department of Orthodontics, Faculty of Dentistry, Cairo University; Egypt; E-mail: yehya3d@gmail.com

Received: November 04, 2020; Accepted: November 10, 2020; Published: November 15, 2020

Anterior open bite $(\mathrm{AOB})$ can be considered one of the most challenging malocclusions to treat. Its complexity arises from the multi-factorial etiology, the involvement of various components and the uncertain stability.

There is abundance of literature on the $\mathrm{AOB}$ classifications, the different etiologies and the possible treatment options. However, most of the articles focus on treatment of $\mathrm{AOB}$ malocclusions, neglecting the deeper search into the components involved into that malocclusion which is essential for proper treatment planning.

AOB can be attributed to skeletal or dental underlying causes/ components, yet it may result from a combination of both. Analysis of these components would allow the orthodontist to depict the underlying causative factor of the presenting AOB malocclusion and customize a proper treatment plan. This is expected to result in a successful outcome, better stability, improved esthetics and more importantly enhance patient satisfaction.

Treatment of AOB should not be viewed as simple as placing a tongue crib for growing individuals, and treating adult patients with posterior segments intrusion or orthognathic surgery. Tongue size \& position, incisal show at rest and on smiling, mandibular plane steepness and lip competence should all be closely monitored before planning what should be done. There is still a need for a detailed systematic analysis of all these components and relating these findings to the possible treatment options to serve as a guide for orthodontists in dealing with such difficult cases. This is currently our area of interest and we are working as a team to establish a schematic approach, aiming to specifically recognize the exact components of $\mathrm{AOB}$ malocclusion and target our treatment accordingly.

\section{Citation:}

Heba E. Akl, Amira A. Aboalnaga, Yehya A. Mostafa (2020) Letter to the Editor - Open Bite Malocclusion: Analysis of the Underlying Components. J Dent Maxillofacial Res Volume 3(3): 1-1. 